\title{
Transcriptional regulatory network for sexual differentiation in fission yeast Juan Mata* ${ }^{* \dagger}$ Anna Wilbrey** and Jürg Bähler*
}

Addresses: *Cancer Research UK Fission Yeast Functional Genomics Group, Wellcome Trust Sanger Institute, Wellcome Trust Genome Campus, Cambridge CB10 1HH, UK. ${ }^{+}$Current address: Department of Biochemistry, University of Cambridge, Downing Site, Cambridge CB2 1QW, UK. *Current address: The Babraham Institute, B501, Babraham Research Campus, Cambridge CB22 3AT, UK.

Correspondence: Juan Mata. Email: jm593@cam.ac.uk

Published: 10 October 2007

Genome Biology 2007, 8:R217 (doi:10.1 186/gb-2007-8-10-r217)

The electronic version of this article is the complete one and can be found online at http://genomebiology.com/2007/8/I0/R2 I7
Received: 17 July 2007

Revised: II September 2007

Accepted: 10 October 2007

(c) 2007 Mata et al; licensee BioMed Central Ltd.

This is an open access article distributed under the terms of the Creative Commons Attribution License (http://creativecommons.org/licenses/by/2.0), which permits unrestricted use, distribution, and reproduction in any medium, provided the original work is properly cited.

\begin{abstract}
Background: Changes in gene expression are hallmarks of cellular differentiation. Sexual differentiation in fission yeast (Schizosaccharomyces pombe) provides a model system for gene expression programs accompanying and driving cellular specialization. The expression of hundreds of genes is modulated in successive waves during meiosis and sporulation in S. pombe, and several known transcription factors are critical for these processes.
\end{abstract}

Results: We used DNA microarrays to investigate meiotic gene regulation by examining transcriptomes after genetic perturbations (gene deletion and/or overexpression) of repl, mei4, atf $I$ and atf $3 I$, which encode known transcription factors controlling sexual differentiation. This analysis reveals target genes at a genome-wide scale and uncovers combinatorial control by Atf I P and Atf3lp. We also studied two transcription factors not previously implicated in sexual differentiation whose meiotic induction depended on Mei4p: Rsv2p induces stress-related genes during spore formation, while Rsvlp represses glucose-metabolism genes. Our data further reveal negative feedback interactions: both Replp and Mei4p not only activate specific gene expression waves (early and middle genes, respectively) but are also required for repression of genes induced in the previous waves (Stel Ip-dependent and early genes, respectively).

Conclusion: These data give insight into regulatory principles controlling the extensive gene expression program driving sexual differentiation and highlight sophisticated interactions and combinatorial control among transcription factors. Besides triggering simultaneous expression of gene waves, transcription factors also repress genes in the previous wave and induce other factors that in turn regulate a subsequent wave. These dependencies ensure an ordered and timely succession of transcriptional waves during cellular differentiation.

\section{Background}

Meiosis and the formation of specialized gametes are fundamental processes of sexual reproduction. Diploid cells of the fission yeast Schizosaccharomyces pombe undergo two mei- otic nuclear divisions to produce four stress-resistant spores in response to environmental stimuli $[1,2]$. This sexual differentiation is accompanied and driven by an extensive gene expression program, during which a large proportion of all 
genes are either induced or repressed [3-5]. We have previously classified the genes that are up-regulated at least fourfold into four major clusters, which represent successive expression waves coinciding with the main biological events of the differentiation process: genes induced in response to environmental changes (starvation and pheromone-induced genes), early genes (pre-meiotic $\mathrm{S}$ phase and recombination), middle genes (meiotic divisions and early steps of spore formation), and late genes (spore maturation) [4].

Posttranscriptional control is involved in regulating mRNA levels during meiosis [6-8]. In addition, transcriptional control is of fundamental importance for sexual differentiation, and several transcription factors are essential for successful meiosis and spore formation. A subset of the genes induced in response to nutritional changes is controlled by the transcription factor Ste11p [3,9], while some early genes are under the control of the Rep1p transcriptional regulator [10-12]. The forkhead-family protein Mei4p controls the expression of several middle genes [13-15], and the basic leucine zipper (bZIP) transcription factors Atf21p and Atf31p control a subset of late genes [4]. It is not known, however, if other transcription factors are involved in this process, and how the activity of the different factors is regulated and coordinated to bring about the orderly succession of transcriptional waves.

Here, we investigate the regulation of meiotic genes by examining the transcriptome of cells deleted for or overexpressing genes encoding transcription factors whose expression is induced during sexual differentiation. Our data highlight the importance of combinatorial control in transcriptional regulation and indicate that the progression of the gene expression waves is achieved by transcriptional cascades and feedback interactions between transcription factors. We also identify two new transcriptional regulators involved in controlling late genes.

\section{Results and discussion}

\section{Rep Ip activates a subset of the early genes}

The Rep1p transcription factor is involved in the regulation of several early genes required for premeiotic $S$ phase and meiotic recombination [10-12]. However, the expression of some early genes is independent of Rep1p [11]. To better understand the role of Rep1p, we sought to systematically identify its target genes by using DNA microarrays to follow gene expression in rep1 $\Delta$ mutant cells undergoing meiosis.

Because good synchrony is important to obtain gene expression profiles of high temporal resolution, we used cells carrying a temperature-sensitive mutation in the meiotic inhibitor Pat1p $[16,17]$. We arrested cells in G1 by removing the nitrogen source from the medium and induced meiosis by shifting the cells to the restrictive temperature. Although pat1induced meiosis is not identical to wild-type meiosis in some respects [18], we have previously shown that the gene expres- sion of early, middle, and late genes is similar in both types of experiments [4].

Deletion of rep 1 had complex effects on the expression of early genes (Figure 1a). About $47 \%$ of early genes were not fully induced in rep1 $\Delta$ cells (full induction was defined as an increase within two-fold of that of wild-type cells at every time point between 1 and 3 hours; see Additional data file for complete lists of Rep1p-dependent and Rep1p-independent genes). However, the effects of rep1 $\Delta$ on gene expression were varied, with some genes being partially induced but often in a delayed fashion compared to wild type. Rep1p-dependent and Rep1p-independent genes showed no clear functional distinction. Both groups were significantly enriched in genes involved in meiotic recombination and meiosis I (GO: o007131 and GO: 0007127, $P<2 \times 10^{-6}$ ), while Rep1pdependent genes were uniquely enriched in genes involved in the mitotic cell-cycle (GO: oooo279, $P<7 \times 10^{-13}$ ). Control of gene expression during early meiosis involves several transcription factors that also function during the mitotic cellcycle (Cdc1op, Res2p and Rep2p), which are likely to cooperate with Rep1p [10]. The large fraction of genes whose expression seems to be independent of Rep1p suggests that additional transcription factors are important for the control of the early genes.

\section{Mei4p regulates the induction of middle genes}

The forkhead-family transcription factor Mei4p is essential for progression through the first meiotic division and has been reported to activate the expression of some middle genes $[14,15]$. It is unclear, however, whether all middle genes are regulated by Mei4p or whether additional transcription factors are involved in their induction. To address this question, we used DNA microarrays to systematically identify Mei4p targets. A dual strategy was applied: first, we compared meiotic time courses of mei4 $\Delta$ cells with wild-type cells; and second, we studied the effect of Mei4p overproduction in vegetative cells.

While deletion of mei4 did not impair the up-regulation of starvation-induced or early genes, the induction of almost all middle and late genes was strongly affected (Figure $1 \mathrm{~b}, \mathrm{c}$ ). Lists of Mei4p-independent and Mei4p-dependent middle genes are presented in Additional data file 9 (Mei4p dependency was defined as an induction at least two-fold lower than that of wild-type cells at any time point between 3 and 7 hours). It is likely that the effect on late genes is indirect, as the expression of some transcription factors regulating late genes is dependent on Mei4p(see below). To distinguish between direct and indirect effects, we took advantage of the finding that overproduction of Mei4p leads to induction of its targets even in vegetative cells $[14,15]$. We cloned the mei4 gene under the control of the regulatable nmt1 promoter [19] and overexpressed mei4 in vegetative cells, in which endogenous mei4 mRNA is almost absent [15]. Mei4p-overproducing cells looked normal 18 hours after induction of the nmt1 

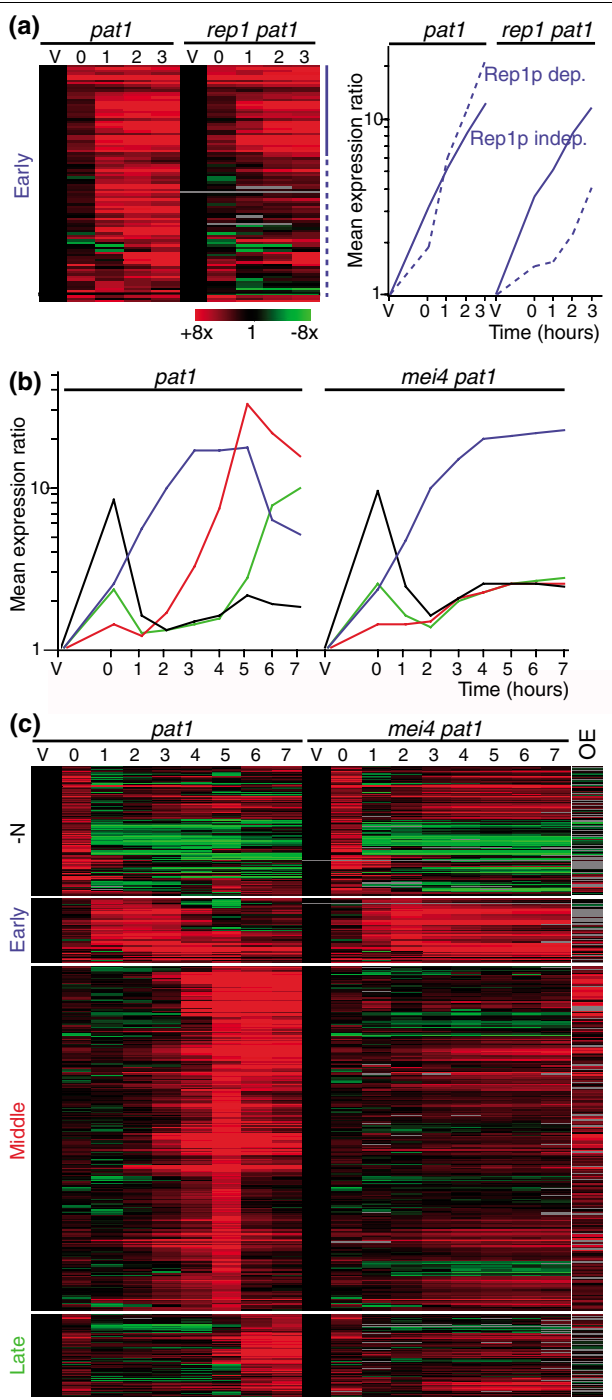

\section{Figure I}

Meiotic gene expression program in rep $/ \Delta$ and mei4 $\Delta$ mutants. (a) Left: hierarchical cluster analysis with columns representing experimental time points and rows representing early genes. Vegetatively growing cells (V) are synchronized in GI by nitrogen removal and enter meiosis by temperature shift at time 0 . The mRNA levels at each time point of pat I (from [4]) and rep I $\Delta$ pat I time courses relative to the levels in vegetative cells are color-coded as indicated at the bottom with missing data in gray. Replp-independent and -dependent genes are indicated at right as drawn-out or dashed blue lines, respectively. Right: average expression profiles of Replp-dependent and Replp-independent genes in wild-type (pat l-synchronized cells from [4]) and rep/ $\Delta$ cells. (b) Average expression profiles of the four main gene clusters upregulated during pat $I$ induced meiosis: nitrogen-starvation response (black), early (blue), middle (red) and late (green), as defined in [4]. Experimental details are as in (a). The $y$-axis shows the expression level at the corresponding time point relative to expression in vegetative cells. Graphs from left to right: pat I cells (from [4]) and mei4 $\Delta$ pat l cells. (c) Hierarchical cluster analysis with columns representing experimental time points and rows representing genes. The mRNA levels at each time point of pat l time courses relative to the levels in vegetative cells are color-coded as indicated in (a). The last column (OE) shows the expression level in cells overproducing Mei4p relative to an empty vector control. The four gene clusters are indicated on the left. promoter (the time point used for microarray analysis). At later time points (24 hours), they appeared smaller than wildtype cells and many cells had lysed. A total of 454 genes were induced at least two-fold following induction of mei4 expression (Figure 1c). This group consisted mostly of middle genes (306), as well 35 genes of the nitrogen response, 19 early genes, and 13 late genes. The remaining genes had not been classified in the original study because they did not pass the four-fold cut-off for gene expression changes relative to vegetative cells [4]. The Mei4p-induced genes included $55 \%$ of all middle genes, but only $16 \%$ of the nitrogen response genes, $19 \%$ of the early genes, and $9 \%$ of the late genes. We noticed that many non-middle genes induced by Mei4p overproduction had complex expression profiles, with several peaks of expression or unusually broad peaks (data not shown). The regulation of these genes is probably under the control of several transcription factors, with Mei4p being responsible for their expression at the time of the meiotic divisions. Of the remaining 81 genes that were induced by Mei4p overproduction but had not been classified, 36 depended on Mei4p for their full induction (as defined above for the mei4s time course; Additional data file 6). Several of the latter showed middle gene-like expression profiles. It is also possible that Mei4p overproduction leads to the artifactual induction of targets of other forkhead transcription factors (Sep1p and Fkh2p) that normally control the periodic expression of a subset of cell cycle-regulated genes [20]. We looked at whether the expression of periodic genes in cluster 1 (which is enriched in Sep1p targets and forkhead-binding sequences) [21] was increased in Mei4p-overproducing cells. The expression of 35 genes of this cluster (out of 94) was induced by Mei4p overproduction. However, 29 of these 35 had been classified as middle genes. Similarly, 9 out of 41 Sep1pdependent periodic targets were induced by Mei4p overexpression, but 8 of them are also middle genes. Therefore, it seems unlikely that Mei4p induces the ectopic expression of Sep1p targets.

Together, the results are consistent with the view that Mei4pdirectly controls the expression of most middle genes, and that the low expression of late genes in a mei4 mutant is an indirect effect. Given that some middle genes (220 out of the 504 Mei4p-dependent middle genes) are not induced by Mei4p overproduction, we cannot rule out the existence of a second factor that activates the expression of middle genes. However, because the expression of almost all middle genes is reduced in a mei44 strain (504 out of 549 show at least a twofold reduction in induction; Figure 1c), we believe this possibility to be unlikely. The strong induction of at least some middle genes, however, may be supported by posttranscriptional mechanisms $[7,22]$.

\section{Control of late genes by Atf 2 I p and Atf I I}

We have previously shown that deletion of either atf21 or atf31 affects the expression of approximately $55 \%$ of the late genes [4]. To better understand how these transcription fac- 
tors function, we overexpressed them in vegetative cells, where they are normally present at low levels [4]. Overproduction of Atf21p resulted in the induction of approximately 25 genes, some of them related to stress processes [23] (Figure 2a, and a complete list is in Additional data file 7). When we took samples for microarray analysis (18 hours after induction of the nmt1 promoter), Atf21p-overproducing cells appeared slightly elongated. At later time points (24 hours), most cells were highly elongated and contained one or more septa (data not shown). Overproduction of Atf31p resulted in the induction of approximately 12 genes, many of which are also induced in response to heat shock [23] (Figure 2b, and a complete list is in Additional data file 7). Note that heat-shock related genes show a highly specific expression pattern during pat1-induced meiosis, with a sharp induction following the initial temperature shift and a quick down-regulation after the meiotic divisions [4]. Despite this finding, atf314 cells respond normally to heat shock (JM, AW and JB, unpublished observation). Atf31p-overproducing cells appeared normal after 18 hours of induction but became elongated after 24 hours (data not shown). However, neither group of genes induced by Atf21p or Atf31p overexpression showed any large overlap with the potential meiotic targets of Atf21p/ Atf31p that we have previously identified [4] (Figure 2a,b, left panels).

We then simultaneously overexpressed both Atf21p and Atf31p. This caused a phenotype similar to that of cells overproducing Atf21p alone, but led to much stronger gene expression changes (325 genes induced at least two-fold), which also showed a highly significant enrichment in the meiotic targets of Atf21p/Atf31p (Figure 2c, and a complete list is in Additional data file 7). Many of the genes that were induced show expression profiles typical of late genes, but had not previously been defined as Atf21p/Atf31p targets (Figure 2c, left). There are two reasons for this difference. First, the definition of Atf21p/Atf31p targets was based on experiments using asynchronous cells, which are less sensitive. Most of the genes not originally defined as Atf21p/Atf31p targets, however, were expressed at slightly lower levels in atf21 $\Delta$ and atf $31 \Delta$ cells (mean expression ratios of 0.8 and 0.91 , respectively) [4]. Second, the four-fold cut-off relative to vegetative cells that we used in the original study means that many of these genes were excluded from the analysis. Therefore, the real number of Atf21p/Atf31p targets is probably considerably higher than the conservative estimate from our previous study [4].

This experiment suggests that Atf21p and Atf31p cooperate to induce the expression of late genes during meiosis, probably by forming a heterodimer. These results highlight the importance of combinatorial control in the regulation of transcriptional programs. The fission yeast genome encodes three additional bZIP family transcription factors besides Atf21p and Atf31p [24-26]. These transcription factors can work as homodimers or heterodimers, creating numerous regulatory

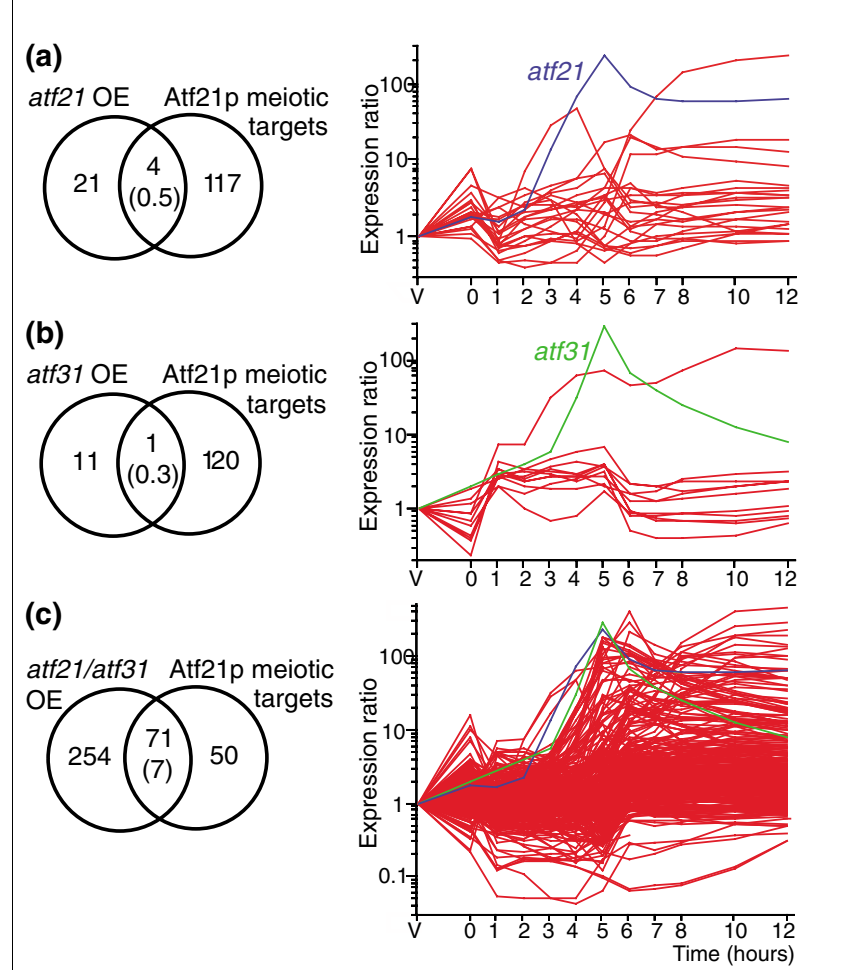

Figure 2

Atf2I $\mathrm{P}$ and Atf3 Ip cooperate to induce expression of meiotic genes. Effects of overproduction of (a) Atf2 Ip, (b) Atf3 Ip, and (c) both Atf2 Ip and Atf3Ip. The Venn diagrams on the left show the overlaps between genes induced by overproduction of the different transcription factors (OE) and potential meiotic Atf2 I p target genes (obtained by analysis of atf 2 I $\Delta$ cells [4]). The numbers in brackets show the overlap between the two lists expected by chance given the sizes of the gene sets considered and the total number of genes. The overlap between Atf 3 Ip-induced genes and meiotic Atf2 Ip targets is not significant, while the overlaps between Atf2I $\mathrm{p}$ and Atf2I $/ \mathrm{Atf} 3 \mathrm{I}$-induced genes and Atf2I $\mathrm{p}$ targets are both significant $\left(P=2.3 \times 10^{-3}\right.$ and $1.5 \times 10^{-62}$, respectively; note, however, that only four Atf $2 \mathrm{I} p$ targets are induced by Atf 2 I p overexpression). The panels on the right show the meiotic expression profiles of genes induced by the overproduction of the corresponding transcription factors (pat Isynchronized cells from [4], experiment and labeling as in Figure I). The profiles of atf2 $I$ and atf $I$ are highlighted in blue and green, respectively.

possibilities. It is likely that control by different combinations of bZIP transcription factors is used by fission yeast to launch specific gene expression programs in response to different environmental or developmental conditions. Combinatorial control by bZIP transcription factors is well known from studies in other organisms (for example, [27,28]).

\section{Two novel transcription factors regulate the induction of late genes}

The expression of many late genes is independent of the Atf21p/Atf31p system [4]. We have previously reported that several genes that potentially encode transcription factors are specifically induced at various stages of sexual differentiation, 
including spore formation, raising the possibility that some of these factors regulate the expression of late genes [4].

We explored the function of $r s v 1$ and $r s v 2$, encoding potential transcription factors with $\mathrm{C}_{2} \mathrm{H} 2$-type zinc fingers that are induced in middle/late meiosis (Figures 3a and 4a). Rsv1p has previously been reported to be required for maintaining viability in stationary phase cells; in particular, Rsvip is required to prevent the loss of viability associated with glucose depletion [29]. Rsv2p has a similar function during stationary phase (L López-Maury, personal communication) and during nitrogen depletion [30]. Deletion of either $r s v 1$ or $r s v 2$ did not affect cell viability or growth of vegetative cells, and rsv1 $\Delta$ and rsv2 $\Delta$ cells were able to mate and form spores of normal morphology that germinated with the same efficiency as that of wild-type spores (data not shown). Despite the lack of a clear meiotic phenotype, we reasoned that the deletions might cause subtle effects on gene expression.

We therefore used DNA microarrays to compare the transcriptome of mutant cells undergoing sexual differentiation with that of wild-type cells. For this experiment, we used homothallic haploid cells without pat1 mutation, which mate to form diploid cells before undergoing sexual differentiation. Because sexual differentiation is less synchronous in these wild-type cells, different meiotic stages can be studied in a single sample [4]. Despite the relative lack of synchrony, these experiments are highly reproducible: only $1.3 \%$ and $1.4 \%$ of the genes showed differences of more than 2-fold between two independent biological replicates of rsv2 and rsv1 experiments, respectively. Most genes were expressed at similar levels in wild-type and rsv2 $\Delta$ cells, showing that meiotic progression and gene expression were not disrupted. However, 39 genes were expressed at lower levels in rsv2 $\Delta$ mutants compared to wild-type cells (reduced at least twofold in two independent repeats; Figure $3 \mathrm{~b}$, and a complete list of genes is in Additional data file 11). Of these genes, 18 had been classified as late genes, and most of the additional 21 genes also showed a late peak of expression (Figure 3c). The latter genes have not been classified as late genes, either because they did not pass the threshold of induction used for the classification, or because they had complex expression patterns containing multiple peaks [4]. A large fraction of the potential Rsv2p targets (17/39) are also induced in several different stress conditions [23]. We conclude that Rsv2p activates the expression of a subset of genes during late meiosis and may contribute to the acquisition of stress resistance by the spore. No obvious regulatory motifs were significantly enriched in the promoters of Rsv2-regulated genes.

Deletion of rsv1 did not cause major changes in gene expression, indicating that meiotic progression and gene expression were normal in the mutant. However, a group of 24 genes were consistently expressed at higher levels compared to wild-type cells (increased at least two-fold in two independent repeats; see Figure $4 \mathrm{~b}$ and Additional data file 10 for a
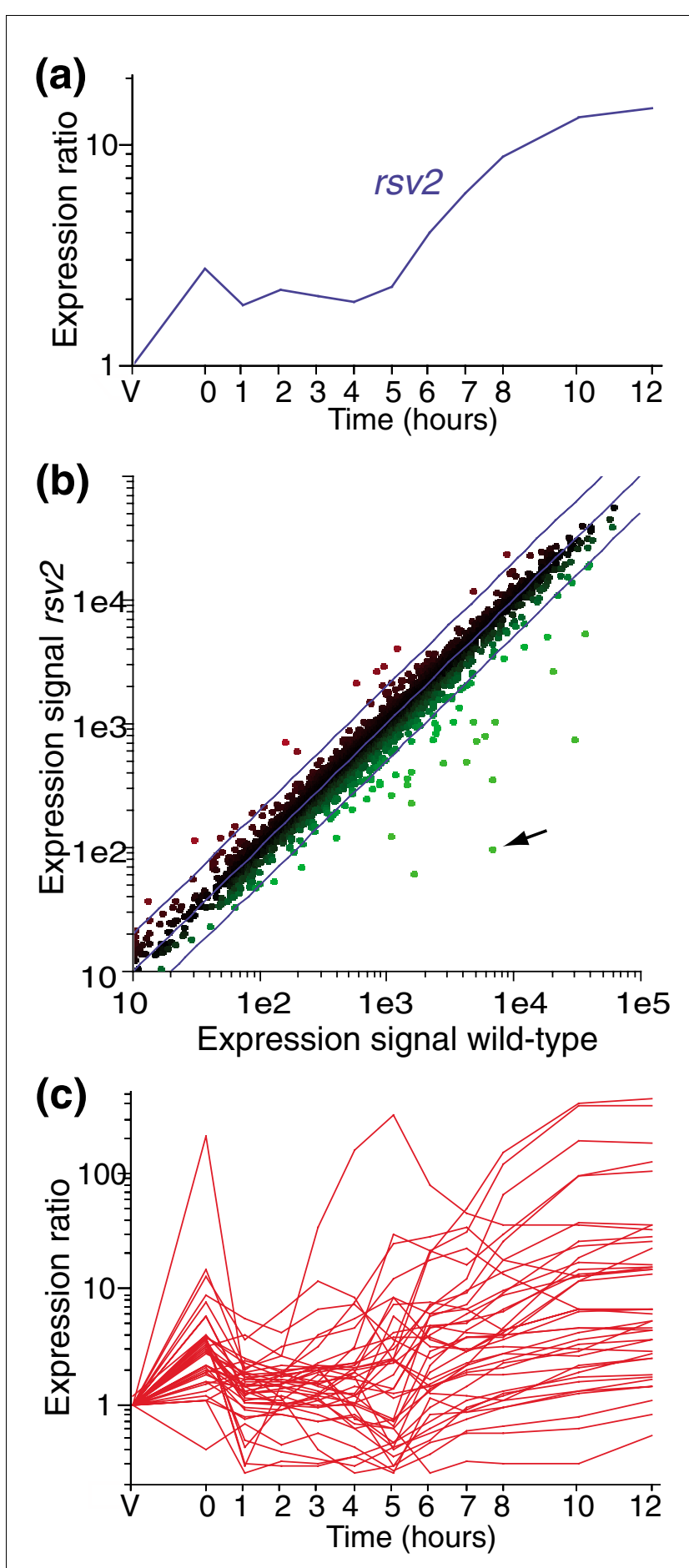

Figure 3

Identification of Rsv2p-dependent genes. (a) Expression profile of rsv2 during meiosis and sporulation (from [4], experiment and labeling as in Figure I). (b) Comparison of gene expression levels between wild-type and $r s v 2 \Delta$ meiotic cells. Genes outside the blue lines differ by more than two-fold in expression levels. rsv2 is indicated by an arrow. (c) Expression profiles of the potential Rsv2p-dependent genes identified in (b) (pat Isynchronized cells from [4]). 

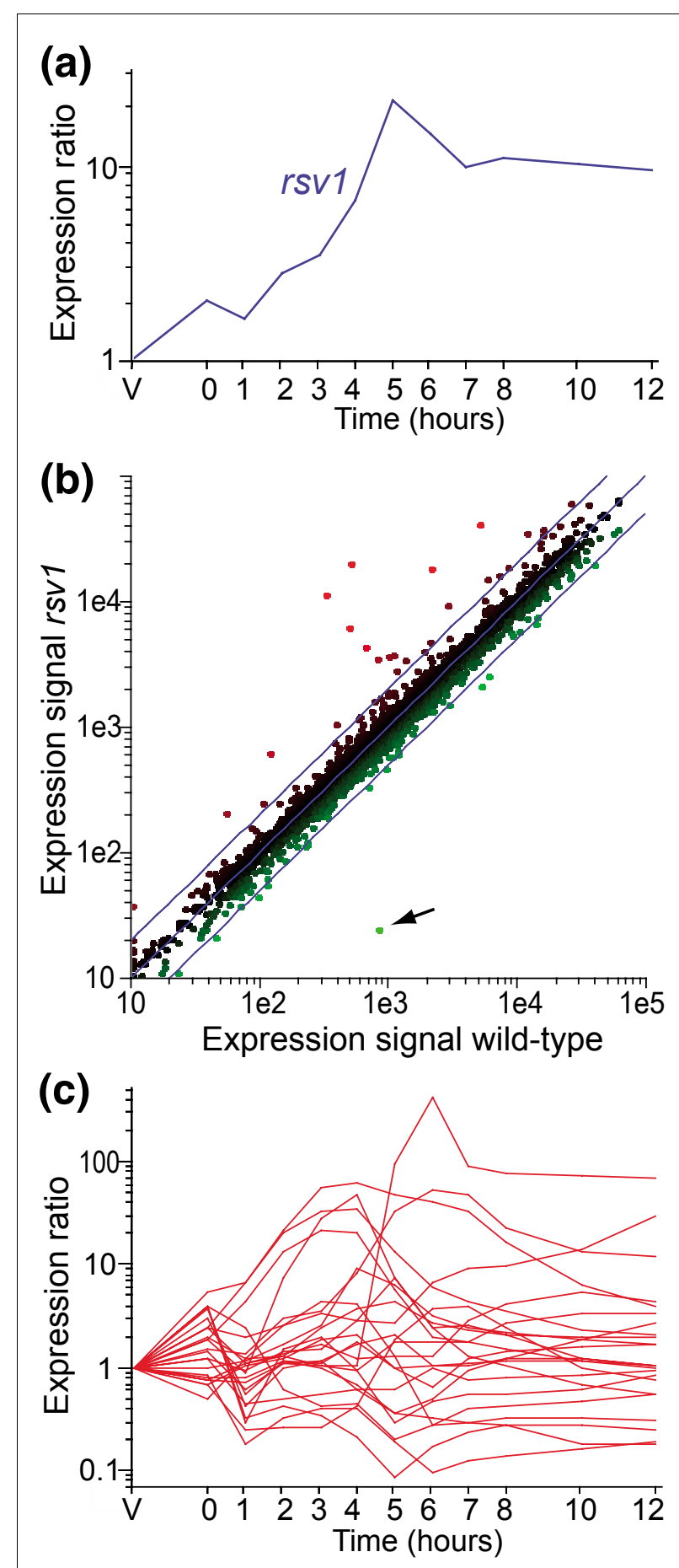

Figure 4

Identification of Rsv Ip-dependent genes. (a) Expression profile of rsv I during meiosis and sporulation (experiment and labeling as in Figure I). (b) Comparison of gene expression levels between wild-type and rsv/ $\Delta$ meiotic cells. Genes outside the blue lines differ by more than two-fold in expression levels. rsvl is indicated by an arrow. (c) Expression profiles of the potential Rsvlp-dependent genes identified in (b) (pat l-synchronized cells from [4]). complete list of genes). This finding suggests that Rsv1p acts as a transcriptional repressor. Many of the induced genes are involved in carbohydrate metabolism, including genes for an inducer of gluconate transport (gti1), four hexose transporters (ght1, ght3, ght4, and ght8), a hexokinase (hsx2), 6-phosphogluconate dehydrogenase (SPB660.16), and a glucose-6phosphate dehydrogenase (SPCC794.01c). The promoters of these genes were enriched in several GC-rich motifs, which might define Rsv1p-binding sites (see Table 1 in Additional data file 5). The potential Rsv1p target genes showed a variety of expression profiles during sexual differentiation in wildtype cells (Figure 4c), but seven of them were early genes, including six genes related to glucose metabolism. Rsv1p is similar to proteins of the Saccharomyces cerevisiae MIP1 family, which includes several transcription factors involved in glucose-repression [31].

Our data show that late genes are induced by at least two separate transcription factor systems (Rsv2p and Atf21p/ Atf31p). Some late genes appear to be independent of both Atf21p/Atf31p and Rsv2p, suggesting that yet other transcription factors are required to activate their expression. However, because our data on Atf21p/Atf31p and Rsv2p targets are based on single time point experiments (which are somewhat less sensitive than time courses), it is possible that all late genes are regulated by these transcription factors and that we have failed to detect a dependency. It is also possible that some mRNAs of late genes are regulated exclusively at the posttranscriptional level.

Regulation by the Atf21p/Atf31p heterodimer appears to be specific for sexual differentiation, while Rsv1p and Rsv2p are used by the cells during both stationary phase and sexual differentiation. Indeed, Rsv1p and Rsv2p appear to regulate related sets of genes during meiosis and stationary phase (JM and $\mathrm{JB}$, unpublished observations). Fission yeast cells thus use the same transcription factors to produce similar physiological responses (quiescence and resistance to environmental stress) in different situations (starvation and differentiation).

\section{Coordination of the meiotic transcriptional program}

The middle genes include several putative transcriptional regulators [4], suggesting a simple model in which expression of Mei4p induces the expression of other transcription factors that, in turn, regulate the late genes. To test this hypothesis, we checked whether the expression of atf21, atf 31, rsv1 and rsv2 was dependent on Mei4p. The atf21, atf31 and rsv1 transcripts were not fully induced in the absence of Mei4p, although atf21 and $r s v 1$ showed a small increase in mei4 $\Delta$ cells (Figure $5 \mathrm{a}-\mathrm{c}$ ). This finding raises the possibility of a twostep activation system, in which only the second step is dependent on Mei4p. The expression of rsv2, on the other hand, is reduced in mei4 $\Delta$ cells at late time points ( 7 hours; Figure $5 \mathrm{~d}$ ), and slightly induced when mei4 is overexpressed (2.1-fold; data not shown). Therefore, it is possible that its 
expression in late meiosis is at least partly under the control of Mei4p.

The alternation between transcriptional waves also requires that each wave is inactivated before the following wave is induced. We found that the reduction in the levels of early genes that normally coincides with the induction of the middle genes did not occur in mei4 $\Delta$ mutants (Figure 1b,c). This effect has been previously observed for a small number of recombination genes [14]. We conclude that in addition to its role in up-regulating middle genes, Mei4p is necessary to switch off the early gene expression wave. This function may be indirect, as we did not observe a down-regulation of early genes when Mei4p is overproduced. The finding that the negative regulator Rsv1p seems to be a Mei4p target (Figure $5 \mathrm{c}$ ) raises the possibility that Mei4p represses the expression of some early genes via Rsvip. However, the inactivation of other early genes, including those involved in meiotic recombination, appears to proceed through a different, yet unknown, mechanism.

Similarly, we noticed that Rep1p, which is involved in the activation of a subset of early genes, is specifically required for the down-regulation of some of the genes induced in response to nitrogen starvation and pheromone signaling following entry into meiosis (Figure 6a,b). This effect is specific to the 'delayed' genes [4], which are enriched in Ste11p targets [3],

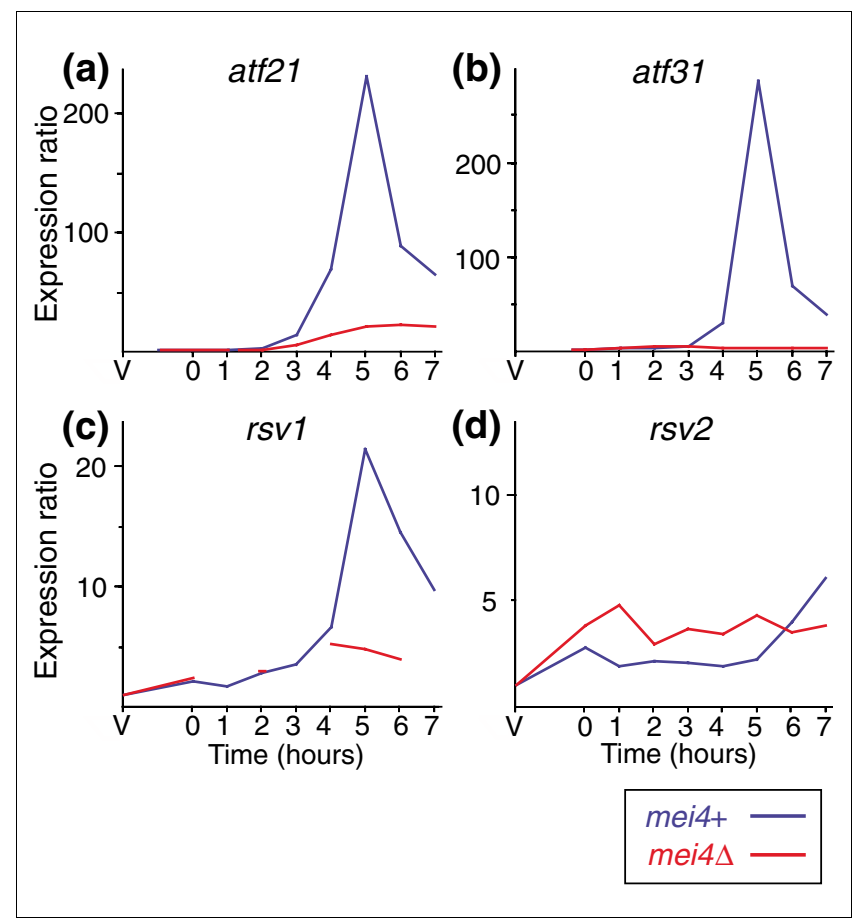

\section{Figure 5}

Transcription factor genes regulated by Mei4p. Expression profiles of genes encoding transcription factors in pat l-synchronized wild-type (blue lines, data from [4]) and mei4 $\Delta$ (red lines) cells: (a) atf2 I, (b) atf3 I, (c) $r s v l$ and (d) rsv2. Experiments and labeling are as in Figure 1. while the down-regulation of other genes induced by nitrogen starvation is unaffected or even stronger in a rep1 $\Delta$ mutant (Figure 6a,b). Although gene expression in wild-type and pat1-induced meiosis is similar for early, middle and late genes [4], the conditions used to trigger synchronous meiosis in pat1 mutants can affect the expression of genes induced by nitrogen starvation. To rule out the possibility that the effects on the expression of delayed genes were an artifact, we checked whether rep1 $\Delta$ mutants had a similar behavior in wild-type meiosis using homothallic cells. Indeed, 'delayed' genes were expressed at higher levels in rep1 $\Delta$ mutants compared to wild-type cells, while 'transient' genes were not affected (Figure 6c).

These results show that the temporal pattern of successive waves of gene expression is at least partly controlled by interactions between transcription factors, in which a transcription factor induces the expression of a wave of genes, while switching off the previous wave and inducing transcription factors that will in turn trigger the next wave (Figure 7).

\section{Conclusion}

We have analyzed the role of six transcription factors in regulating the gene expression program of different stages of sexual developmental in fission yeast. Five of these factors work as transcriptional activators, while one of them (Rsv1p) is a transcriptional repressor. In addition to a global identification of potential target genes, our results give insight into regulatory circuits that coordinate different transcriptional waves of this complex gene expression program. Two main principles are highlighted by this work (Figure 7). First, dependence between transcriptional waves is achieved by positive and negative interactions between transcription factors. Rep1p, which is transcriptionally induced by the master regulator for the pheromone response Ste11p [3,12], activates the expression of early genes but is also essential for the repression of pheromone-response genes. Similarly, Mei4p activates the expression of middle genes and is also essential for the repression of the early genes, and for the induction of genes encoding transcription factors that in turn activate the expression of late genes. These dependencies between transcription factors ensure the ordered, uni-directional succession of transcriptional waves. Second, the importance of combinatorial control: overexpression of Atf21p, Atf31p, or Atf21p/Atf31p leads to the activation of unique sets of targets. The use of different combinations of transcription factors enhances the regulatory options of cells to express specific gene sets in response to a variety of situations.

\section{Materials and methods Yeast methods and experimental design}

Induction of meiosis using pat1 mutations was carried out exactly as in our previous study [4]. Briefly, for the mei44 deletion time course, pat1-114/pat1-114 mei44::ura4 ${ }^{+}$/ 


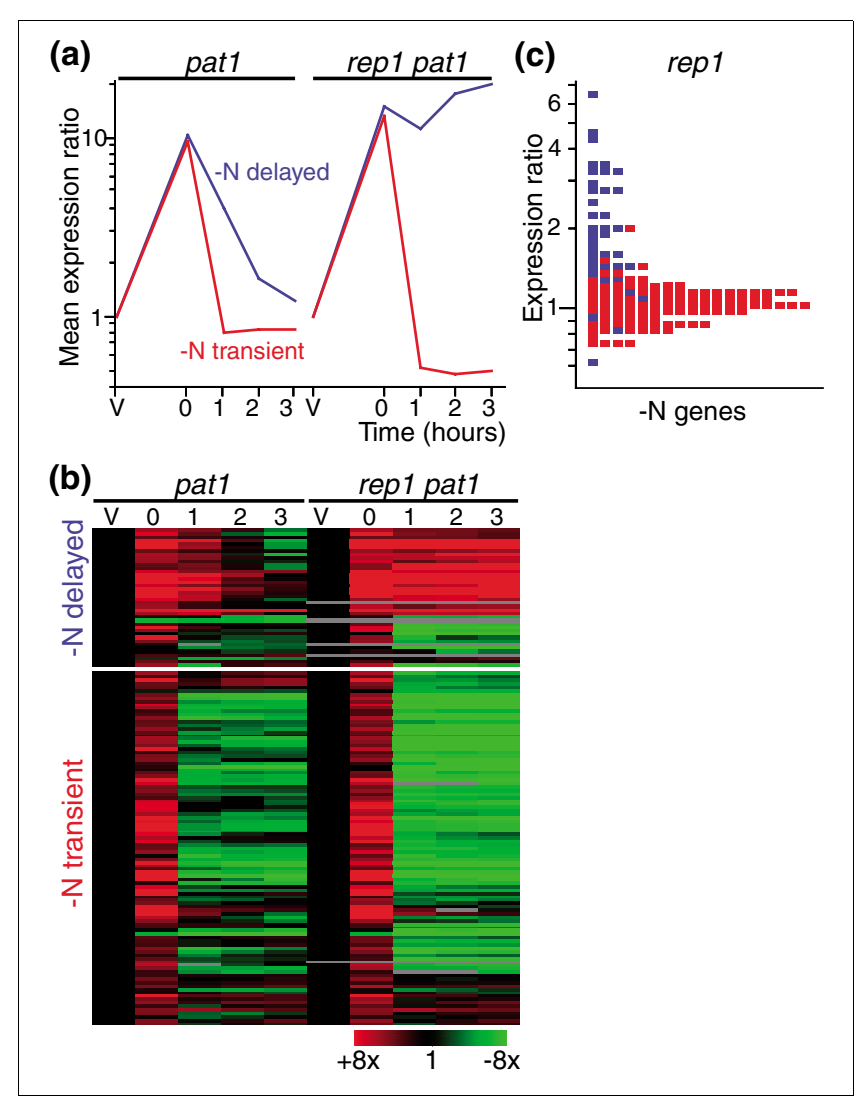

Figure 6

Replp is required to down-regulate a subset of nitrogen starvationinduced genes. (a) Average expression profiles in wild-type (pat lsynchronized cells from [4]) and rep I $\Delta$ cells of two subclusters of genes induced in response to nitrogen starvation: delayed (blue) and transient (red), as defined by [4]. (b) Hierarchical cluster analysis of the two gene clusters shown in (a), with columns representing experimental time points and rows representing genes. The mRNA levels at each time point of pat $I$ time courses relative to the levels in vegetative cells are color-coded as indicated at the bottom with missing data in gray. Labeling is as in Figure I. (c) Histogram showing the gene expression levels of rep $I \Delta$ relative to wild-type meiotic cells. The two clusters are colored as in (a).

mei44::ura4+ura4-D18/ura4-D18 ade6-M21o/ade6-M216 $h$ - $/ h$ - diploid cells were grown in Edinburgh minimal medium containing $2 \%$ glucose (EMM) plus $0.5 \% \mathrm{NH}_{4} \mathrm{Cl}$, and then resuspended in EMM without $\mathrm{NH}_{4} \mathrm{Cl}$ (EMM-N) and incubated for $14 \mathrm{~h}$ at $25^{\circ} \mathrm{C}$. Meiosis was started by shifting the cells to $34^{\circ} \mathrm{C}$ in the presence of $0.05 \% \mathrm{NH}_{4} \mathrm{Cl}$. The rep $1 \Delta$ deletion time course was carried out in a similar way using pat1-114/ pat1-114 rep14::ura4+/rep14::ura4 ${ }^{+}$ura4-D18/ura4-D18 ade6-M210/ade6-M216 $h+/ h+$ diploid cells. For pat1 time courses, RNA extracted from each time point was compared to a reference RNA prepared from pat1-114/pat-114 cells treated as described above to induce meiosis. The reference consisted of equal amounts of RNA extracted from vegetative cells and cells at o, 1, 2, 3, 4, 5, 6, 7, 8, 10 and 12 hours after the temperature shift. The expression ratios at each time point were normalized to those of vegetative cells of the corresponding strain.

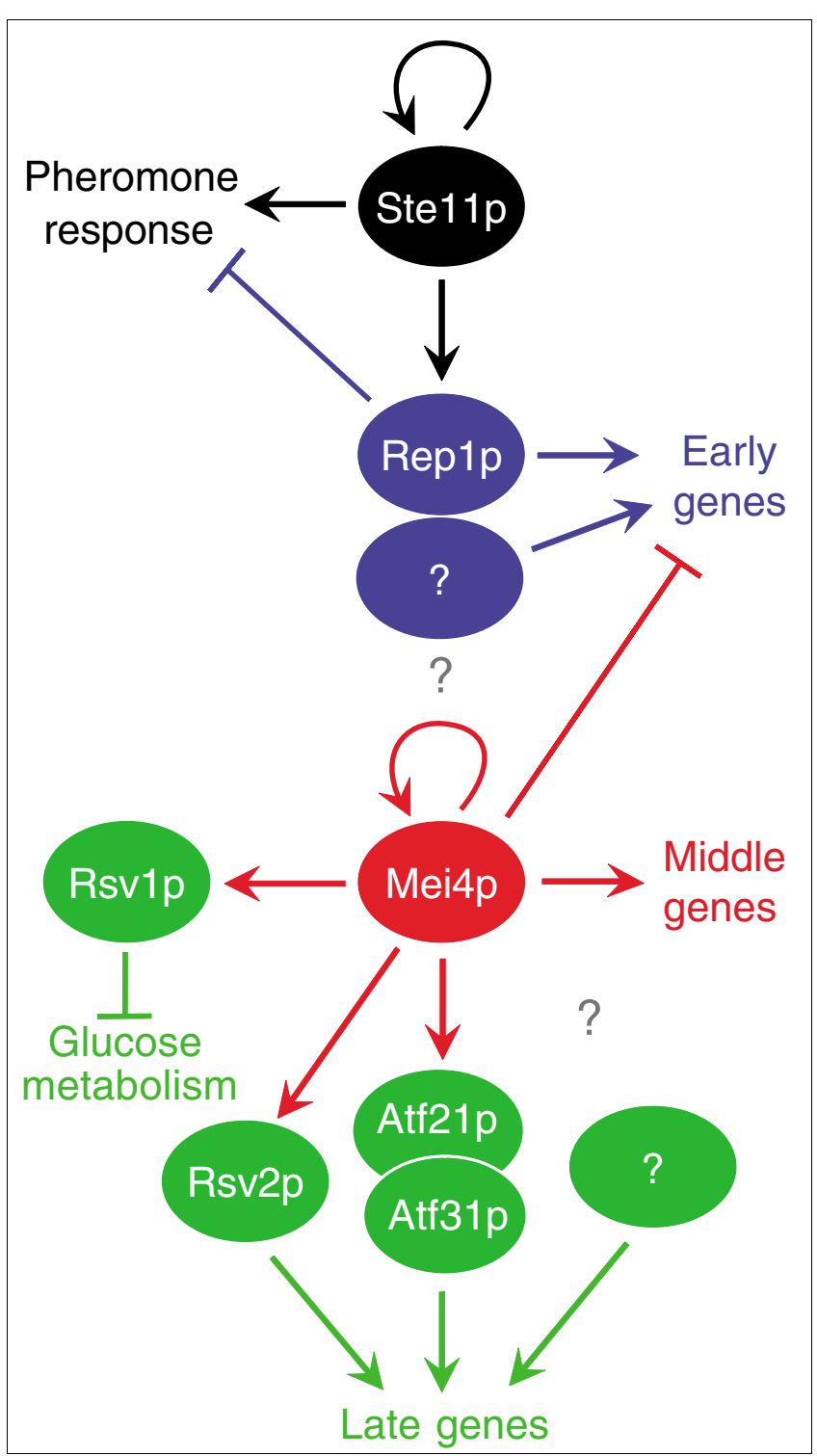

Figure 7

Model for transcriptional regulatory network controlling meiosis and sporulation. Arrows indicate activation and cross bars indicate repression. The colors reflect the different stages of sexual differentiation as in Figure I. Mei4p controls transcription of its own gene in a positive feedback [14], but no other transcription factors have been identified that trigger the initial induction of mei4. This activation could be achieved at the posttranscriptional level [7]. Refer to the main text for further details on interactions.

We deleted rsv1 and rvs2 in a homothallic h9o background using the one-step PCR method [32]. Meiosis in the mutants was induced by incubating cells in EMM containing $0.5 \%$ glucose without $\mathrm{NH}_{4} \mathrm{Cl}$ at $28^{\circ} \mathrm{C}$. Samples were harvested after 15 h. Wild-type h9o cells treated in parallel in exactly the same way were used as a reference. For overexpression experiments, the coding sequences of the mei4, atf21 and atf 31 genes were amplified by PCR and cloned in the $\mathrm{PREP}_{3} \mathrm{X}$ vector [33], which contains the inducible nmt1 promoter and a 
LEU2 selectable marker. For the co-overexpression experiment, atf31 was cloned into pREP4X (also under nmt1 control, but with an ura4 selectable marker). Single plasmids were transformed into a leu1-32 $h$ - strain, and both plasmids were co-transformed into leu1-32 ura4-D18 $h$ - cells. To induce the nmt1 promoter, cells were grown in EMM containing $15 \mu \mathrm{M}$ thiamine, washed three times in EMM, and incubated at $32^{\circ} \mathrm{C}$ for $18 \mathrm{~h}$. In every experiment, RNA extracted from cells overexpressing a particular transcription factor was compared with RNA from cells transformed with empty vectors that were treated in exactly the same way to induce the nmt1 promoter.

\section{Microarray experiments}

RNA preparation, labeling, microarray production, and data processing were performed as described [34]. Microarrays were scanned with a Genepix $4000 B$ scanner and analyzed with GenePix software (Molecular Devices, Sunnyvale, CA, USA). Hierarchical clustering, visualization, and regulatory motif searches were done with GeneSpring (Agilent, Santa Clara, CA, USA). The significance of the overlaps between gene lists was determined assuming that the overlap between random groups follows a hypergeometric distribution. All processed and normalized data are available from our website [35], and the entire raw data sets have been deposited in Array Express [36] with accession numbers E-TABM-298, ETABM-299, E-TABM-300 and E-TABM-301. Complete normalized data sets are also available in Additional data files 14. Microarray experiments of $r s v 1 \Delta$ and $r s v 2 \Delta$ deletions and overexpression of Atf21p and Atf31p transcription factors were done in duplicate (independent biological repeats including a dye swap). The RNA from the Mei4p overexpression experiment was hybridized in duplicate (technical repeat, including a dye swap). The mei4 $\Delta$ and rep1 $\Delta$ time courses and the wild-type rep1 $\Delta$ experiment were carried out once.

\section{Validation of results}

We compared our data with Northern-based experiments of gene expression in rep1 $\Delta$ and mei4 $\Delta$ backgrounds. The microarray results were similar to published data for 8/10 genes in rep1 $\Delta$ and 37/42 genes in mei4 $\Delta$ (see Tables 2 and 3 in Additional data file 5).

\section{Abbreviations}

bZIP, basic leucine zipper; EMM, Edinburgh minimal medium.

\section{Authors' contributions}

JM and AW carried out the experiments. JM and JB conceived the study, analyzed the data and co-wrote the paper. All authors read and approved the final manuscript.

\section{Additional data files}

The following additional data are available with the online version of this paper. Additional data file 1 includes the complete normalized dataset for mei44 pat1 and pat1 time courses. Additional data file 2 includes the complete normalized dataset for rep1 1 pat1 and pat1 time courses. Additional data file 3 includes the complete normalized dataset for $r s v 1 \Delta$ and rsv2 $\Delta$ experiments. Additional data file 4 includes the complete normalized dataset for Atf21p, Atf31p, Atf21p/ Atf31p and Mei4p overexpression experiments. Additional data file 5 includes additional tables. Table 1: Potential regulatory motifs in the promoters of Rsv1p-regulated genes. Table 2: Effects of rep1 $\Delta$ on meiotic transcription: comparison with published data. Table 3: Effects of mei4 $\Delta$ on meiotic transcription; comparison with published data. Additional data file 6 lists middle genes classified into three groups according to the effect of mei4 $\Delta$ and Mei4p overexpression on their expression. Additional data file 7 lists genes induced by Atf21p, Atf31p and Atf21p/Atf31p overexpression and genes induced by Atf21p/Atf31p overexpression that are also reduced in atf21 4 meiotic cells. Additional data file 8 lists genes induced by Mei4p overexpression, classified according to their expression profiles. Additional data file 9 lists Mei4pdependent and Mei4p-independent middle genes based on the mei4 $\Delta$ time course experiment. Additional data file 10 includes a complete list of potential Rsv1p targets. Additional data file 11 includes a complete list of potential Rsv2p targets. Additional data file 12 lists Rep1p-dependent and Rep1pindependent early genes based on the rep1 $\Delta$ time course experiment.

\section{Acknowledgements}

We thank Fausto Arellano for help with strain construction and microarray processing, Samuel Marguerat, Luis López-Maury and Daniel Lackner for comments on the manuscript, and the Sanger microarray facility for array printing. This work was supported by Cancer Research UK (CUK) Grant No. C9546/A65I7.

\section{References}

I. Shimoda C, Nakamura T: Control of late meiosis and ascospore formation. In The Molecular Biology of Schizosaccharomyces pombe Edited by: Egel R. Heidelberg: Springer-Verlag; 2004:3 I I-327.

2. Yamamoto M: Initiation of meiosis. In The Molecular Biology of Schizosaccharomyces pombe Edited by: Egel R. Heidelberg: SpringerVerlag; 2004:297-310.

3. Mata J, Bähler J: Global roles of Ste I Ip, cell type, and pheromone in the control of gene expression during early sexual differentiation in fission yeast. Proc Natl Acad Sci USA 2006, 103:15517-15522.

4. Mata J, Lyne R, Burns G, Bähler J: The transcriptional program of meiosis and sporulation in fission yeast. Nat Genet 2002, 32: $143-147$

5. Xue-Franzen Y, Kjaerulff S, Holmberg C, Wright A, Nielsen O: Genomewide identification of pheromone-targeted transcription in fission yeast. BMC Genomics 2006, 7:303.

6. Averbeck N, Sunder S, Sample N, Wise JA, Leatherwood J: Negative control contributes to an extensive program of meiotic splicing in fission yeast. Mol Cell 2005, I 8:49I-498.

7. Harigaya $Y$, Tanaka $H$, Yamanaka S, Tanaka K, Watanabe $Y$, Tsutsumi C, Chikashige $Y$, Hiraoka Y, Yamashita A, Yamamoto M: Selective elimination of messenger RNA prevents an incidence of untimely meiosis. Nature 2006, 442:45-50. 
8. Watanabe $Y$, Yamamoto M: S. pombe mei2+ encodes an RNAbinding protein essential for premeiotic DNA synthesis and meiosis I, which cooperates with a novel RNA species meiRNA. Cell 1994, 78:487-498.

9. Sugimoto A, lino $Y$, Maeda T, Watanabe $Y$, Yamamoto M: Schizosaccharomyces pombe ste II+ encodes a transcription factor with an HMG motif that is a critical regulator of sexual development. Genes Dev 1991, 5:1990-1999.

10. Cunliffe L, White S, Mclnerny CJ: DSCI-MCB regulation of meiotic transcription in Schizosaccharomyces pombe. Mol Genet Genomics 2004, 27 I:60-7I.

II. Ding R, Smith GR: Global control of meiotic recombination genes by Schizosaccharomyces pombe recl6 (repl). Mol Gen Genet 1998, 258:663-670.

12. Sugiyama A, Tanaka K, Okazaki K, Nojima H, Okayama H: A zinc finger protein controls the onset of premeiotic DNA synthesis of fission yeast in a Mei2-independent cascade. EMBO J 1994, | 3: $188 \mid-1887$.

13. Watanabe T, Miyashita K, Saito TT, Yoneki T, Kakihara Y, Nabeshima $\mathrm{K}$, Kishi YA, Shimoda C, Nojima H: Comprehensive isolation of meiosis-specific genes identifies novel proteins and unusual non-coding transcripts in Schizosaccharomyces pombe. Nucleic Acids Res 2001, 29:2327-2337.

14. Abe H, Shimoda C: Autoregulated expression of Schizosaccharomyces pombe meiosis-specific transcription factor Mei4 and a genome-wide search for its target genes. Genetics 2000, 154:1497-1508.

15. Horie S, Watanabe $\mathrm{Y}$, Tanaka K, Nishiwaki S, Fujioka $\mathrm{H}$, Abe $\mathrm{H}$, Yamamoto M, Shimoda C: The Schizosaccharomyces pombe mei4+ gene encodes a meiosis-specific transcription factor containing a forkhead DNA-binding domain. Mol Cell Biol I998, I8:21|8-2|29.

16. lino $Y$, Yamamoto $M$ : Negative control for the initiation of meiosis in Schizosaccharomyces pombe. Proc Natl Acad Sci USA 1985, 82:2447-245।.

17. McLeod M, Beach D: Homology between the ran I+ gene of fission yeast and protein kinases. EMBO J 1986, 5:3665-367I.

18. Yamamoto A, Hiraoka Y: Monopolar spindle attachment of sister chromatids is ensured by two distinct mechanisms at the first meiotic division in fission yeast. EMBO J 2003, 22:2284-2296

19. Maundrell K: Thiamine-repressible expression vectors pREP and pRIP for fission yeast. Gene 1993, 123:127-130.

20. Bähler J: Cell-cycle control of gene expression in budding and fission yeast. Annu Rev Genet 2005, 39:69-94.

21. Rustici G, Mata J, Kivinen K, Lio P, Penkett CJ, Burns G, Hayles J, Brazma A, Nurse $P$, Bähler J: Periodic gene expression program of the fission yeast cell cycle. Nat Genet 2004, 36:809-8I7.

22. Mata J, Marguerat S, Bähler J: Post-transcriptional control of gene expression: a genome-wide perspective. Trends Biochem Sci 2005, 30:506-5।4

23. Chen D, Toone WM, Mata J, Lyne R, Burns G, Kivinen K, Brazma A, Jones N, Bähler J: Global transcriptional responses of fission yeast to environmental stress. Mol Biol Cell 2003, I 4:2 I4-229.

24. Harrison C, Katayama S, Dhut S, Chen D, Jones N, Bähler J, Toda T: SCF(Pofl)-ubiquitin and its target Zip I transcription factor mediate cadmium response in fission yeast. EMBO J 2005, 24:599-610.

25. Takeda T, Toda T, Kominami K, Kohnosu A, Yanagida M, Jones N: Schizosaccharomyces pombe atfl+ encodes a transcription factor required for sexual development and entry into stationary phase. EMBO J 1995, 14:6193-6208.

26. Watanabe $Y$, Yamamoto M: Schizosaccharomyces pombe pcrl+ encodes a CREB/ATF protein involved in regulation of gene expression for sexual development. Mol Cell Biol 1996, 16:704-7II.

27. Newman JR, Keating AE: Comprehensive identification of human bZIP interactions with coiled-coil arrays. Science 2003, 300:2097-2101.

28. Weltmeier F, Ehlert A, Mayer CS, Dietrich K, Wang X, Schutze K, Alonso R, Harter K, Vicente-Carbajosa J, Droge-Laser W: Combinatorial control of Arabidopsis proline dehydrogenase transcription by specific heterodimerisation of bZIP transcription factors. $E M B O$ o 2006, 25:3|33-3|43.

29. Hao Z, Furunobu $A$, Nagata $A$, Okayama $\mathrm{H}$ : A zinc finger protein required for stationary phase viability in fission yeast. J Cell Sci 1997, I I 0:2557-2566.

30. Shimanuki M, Chung SY, Chikashige Y, Kawasaki Y, Uehara L, Tsut- sumi C, Hatanaka M, Hiraoka Y, Nagao K, Yanagida M: Two-step, extensive alterations in the transcriptome from $\mathbf{~} 0$ arrest to cell division in Schizosaccharomyces pombe. Genes Cells 2007, I 2:677-692.

31. Lutfiyya LL, lyer VR, DeRisi J, DeVit MJ, Brown PO, Johnston M: Characterization of three related glucose repressors and genes they regulate in Saccharomyces cerevisiae. Genetics 1998, 150:1377-1391.

32. Bähler J, Wu JQ, Longtine MS, Shah NG, McKenzie A 3rd, Steever AB, Wach A, Philippsen P, Pringle JR: Heterologous modules for efficient and versatile PCR-based gene targeting in Schizosaccharomyces pombe. Yeast 1998, 1 4:943-951.

33. Forsburg SL, Sherman DA: General purpose tagging vectors for fission yeast. Gene 1997, 191:191-195.

34. Lyne R, Burns G, Mata J, Penkett CJ, Rustici G, Chen D, Langford C Vetrie $D$, Bähler J: Whole-genome microarrays of fission yeast: characteristics, accuracy, reproducibility, and processing of array data. BMC Genomics 2003, 4:27.

35. Fission Yeast Functional Genomics [http://www.sanger.ac.uk/ PostGenomics/S_pombe/]

36. Array Express [http://www.ebi.ac.uk/arrayexpress/] 